CORRECTION

\title{
Correction: Dutch Pharmacogenetics Working Group (DPWG) guideline for the gene-drug interaction between CYP2D6 and opioids (codeine, tramadol and oxycodone)
}

Maja Matic, Marga Nijenhuis (D), Bianca Soree, Nienke J. de Boer-Veger, Anne-Marie Buunk, Elisa J. F. Houwink, Hans Mulder, Gerard A. P. J. M. Rongen, Jan van der Weide, Bob Wilffert, Jesse J. Swen, Henk-Jan Guchelaar, Vera H. M. Deneer and Ron H. N. van Schaik

(c) The Author(s), under exclusive licence to European Society of Human Genetics 2021

European Journal of Human Genetics (2022) 30:1196; https://doi.org/10.1038/s41431-021-00969-9

Correction to: European Journal of Human Genetics https://doi.org/ 10.1038/s41431-021-00920-y, published online 15 July 2021

The Data statement was partly wrong and should have read as below.

\section{DATA AVAILABILITY}

All data and material are either included in the Supplementary information or publicly available (i.e., the published articles,
PubMed). The guidelines and background information are available on the website of the Royal Dutch Pharmacists Association (KNMP) (Pharmacogenetic Recommendations. Available from: https://www.knmp.nl/). The guidelines and background information will be available on PharmGKB.org. 